\title{
OPTICAL TRANSITIONS IN PROTON TRANSFER SYSTEMS
}

\author{
(Presented by K. K. Rebane)
}

The low-temperature narrow-line spectra of optical impurity centres doped substitutionally into carboxylic acid crystals carry information about the proton structure of the environment. This fact has been used in benzoic acid crystals containing thio- and selenoindigo to measure the tautomerization dynamics of the acid dimers and to determine the tunneling matrix element of the double well potential governing this proton transfer.

\section{Introduction}

Two-level systems, double-well potentials, and tunneling systems have been invoked to explain the optical properties of impurity centers in glasses. They are used to rationalize the mechanisms of both the burning and the broadening of spectral holes produced by frequency selective laser irradiation in the inhomogeneous absorption line of the optical center $\left[{ }^{1,2}\right]$. Yet the exact physical nature of the tunneling system is obscure: in hydrogen-bonded organic glasses large deuteration effects have been found pointing to the role of protons of the hydrogen bonds $[3,4]$, but any detailed study is hindered by the wide distribution of two-level systems coupled to the optical center, which makes it difficult if not impossible to uncover the behaviour of a specific center. The detailed study of well-defined tunneling systems as can be observed in hydrogen-bonded crystals is interesting in this regard. In a more general context these systems can be used as models for the study at low temperatures of the interplay of tunneling and relaxation in a tunneling system coupled to a heat bath, a subject of much current theoretical interest $\left[{ }^{5-8}\right]$. These systems are also relevant for the description of the transition from quantum motion at low temperatures to classical transport at higher temperatures and provide models for the study of hydrogen transfer reactions along hydrogen bonds.

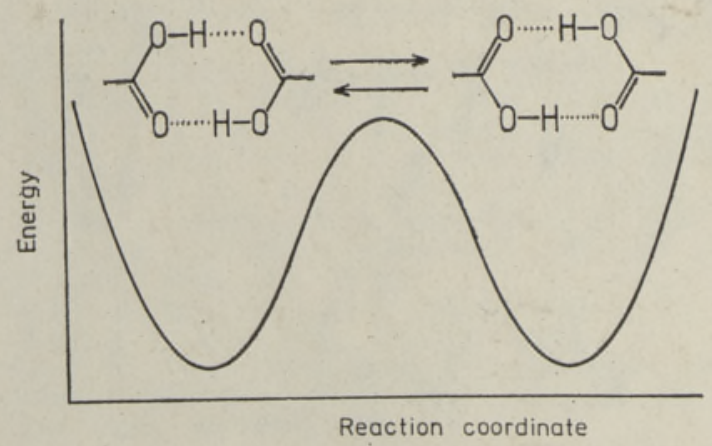

Fig. 1, Tautomerization of a symmetric carboxylic acid dimer. 
Our model system is shown in Fig. 1. Carboxylic acids form dimers linked by two hydrogen bonds and there exist two tautomer forms separated by a potential barrier, which interconvert by a concerted twoproton transfer along the hydrogen bonds. For an isolated symmetric dimer the tautomerization is therefore governed by a symmetric doublewell potential and one of the questions that arises concerns the value of the tunneling splitting in such a potential. In the tautomerization reaction not only the protons move (by ca. $0.7 \AA$ ) but also to minor extent the other atoms of the skeleton: $\mathrm{C}-\mathrm{O}$ double and single bonds, for example, are interchanged and the corresponding bond distances vary by $0.1 \AA$. It follows that the reaction coordinate is not simply given by the position of the hydrogen atoms and that the value of the tunneling splitting is lowered, reflecting the weight of the heavier skeleton coordinates involved in the reaction $\left[{ }^{9-11}\right]$. In a condensed phase environment this splitting is further lowered by the coupling to the bath coordinates and in addition the tunneling oscillation is damped by this coupling $\left[{ }^{12}\right]$. The coupling to the bath coordinates leads to a structural relaxation of the environment around each of the tautomer forms, but in a low temperature solid this relaxation cannot fully occur on the timescale of the tunneling oscillation: the two tautomers become distinct and have different energies and at low temperatures the system is trapped in the most stable form, as the energy difference of the two tautomers is in general large as compared to the tunneling splitting.

In a pure crystal, therefore, the two tautomers are distinguishable and depending upon their energy difference and the temperature both forms will be more or less populated. At finite temperatures the protons are not in an ordered configuration and the question arises whether this disorder is static or dynamic on the timescale of an optical experiment for example. This timescale is given by the rate of tautomer interconversion: nuclear magnetic resonance and inelastic neutron scattering techniques have recently been used to determine its value in a number of carboxylic acid crystals at higher temperatures $(T>100 \mathrm{~K})$ when the process occurs by thermally activated barrier crossing $\left[{ }^{13-19}\right]$. At lower temperatures direct transitions between the quantum states of the system are thought to dominate, but their rate could not be determined because at low temperatures only the most stable tautomer is populated, the protons are ordered, and all dynamics is frozen out.

The object of our work is to show how optical methods, using a dye molecule as a probe of the environment, provide information on the tunneling level structure and the dynamics of the system at very lowtemperatures. In this paper I shall summarize the results of recent work done in collaboration with R. M. Hochstrasser and G. R. Holtom in Philadelphia and with M. Pierre and Ch. Rambaud in Grenoble $\left[{ }^{20-26}\right]$.

\section{Optical transitions of a dye coupled to a carbexylic acid dimer}

The energy difference of the two tautomers of a symmetric carboxylic acid dimer in the solid state is - as explained above - determined by the coupling to, and the static structure of the environment. In a crystal the molecular packing determines the asymmetry of the double-well potential; frequently all dimers are symmetry related and the tautomerization is described by a unique potential function. In order to change the value of the asymmetry it is possible to apply external electric or strain fields, but in general these perturbations lead to insignificant changes of the potential only. Using different compounds with different crystal structures it is, of course, possible to realize a variety of asymmetries, but a more versatile and easier way to alter locally the potential func- 
tion of selected dimers in the crystal is to dope the crystal with suitable impurity molecules. When a carboxylic acid dimer is replaced substitutionally by an impurity the tautomerization potential of neighboring dimers is perturbed and for these dimers the asymmetries of the doublewell potential span a set of well-defined descrete values. The distribution of asymmetries depends not only upon the chemical nature of the impurity but also upon the electronic state. For studies at low temperatures a limited number of dimers will be relevant only:

a) dimers for which the asymmetry of the potential has been reduced to a value below the asymmetry of the bulk material and also sufficiently small, such that both tautomers are thermally populated;

b) dimers for which the asymmetry of the double-well potential is reversed when the impurity is promoted into an electronically excited state, such that in an optical transition of the impurity the unstable form of the neighboring acid dimer is populated.

\section{Time resolved fluorescence measurements of the tautomerization dynamics}

Fig. 2 illustrates schematically how the dynamics of tautomerization can be monitored in case b) mentioned above, using time resolved measurements of the emission of the dye impurity subsequent to frequency selective pulsed excitation. The labelling of the different levels is given in the figure; the decay rates of the $|1, \alpha\rangle$ and $|1, \beta\rangle$ excited states are denoted by $k_{1 \alpha}$ and $k_{1 \beta}$, respectively, and $k_{\alpha \beta}$ represents the rate of tautomerization $|1, \alpha\rangle \rightarrow|1, \beta\rangle$. With these notations the intensities $I_{1}(t)$ and $I_{2}(t)$ of the $|1, \alpha\rangle \rightarrow|0, \alpha\rangle$ and $|1, \beta\rangle \rightarrow|0, \beta\rangle$ transitions subsequent to pulsed excitation at the $|0, \alpha\rangle \rightarrow|1, \alpha\rangle$ transition frequency is given by:

$$
\begin{aligned}
& I_{1}(t)=A \cdot \exp \left(-k_{1 \alpha} \cdot t\right) \\
& I_{2}(t)=A \cdot k_{\alpha \beta} /\left(k_{1 \alpha}-k_{1 \beta}\right) \cdot\left\{\exp \left(-k_{1 \beta} \cdot t\right)-\exp \left(-k_{1 \alpha} \cdot t\right)\right\}
\end{aligned}
$$

The ratio of the time integrated emissions is:

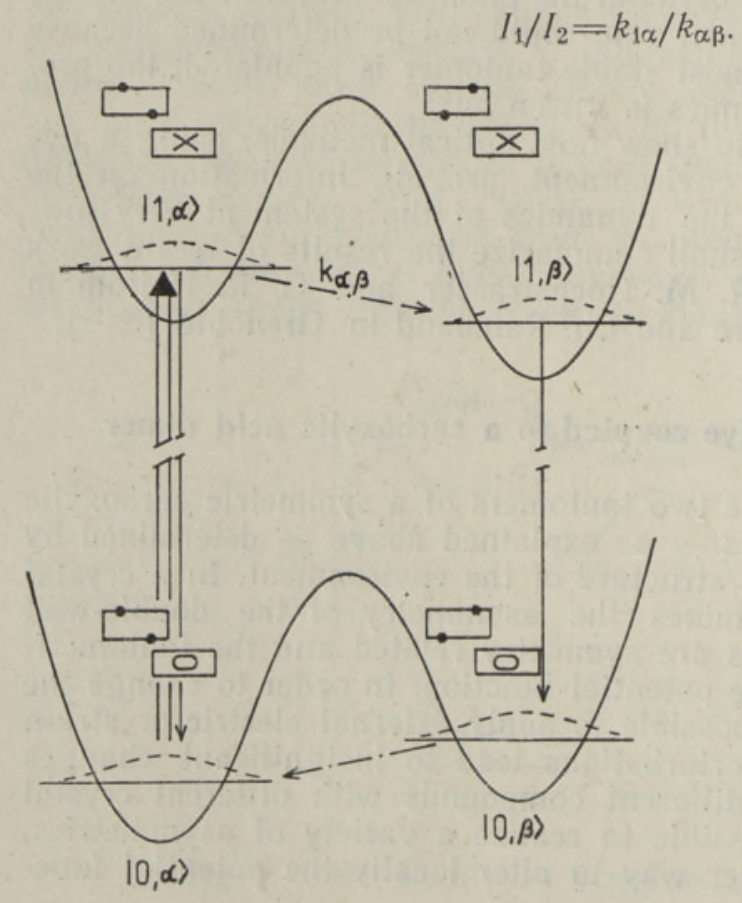

Fig. 2. Schematic representation of the double-well tautomerization potential of an acid dimer coupled to a dye molecule. The wavefunctions are indicated as broken lines. The asymmetry of the doublewell potential is reversed when the dye molecule is excited. The rate of tautomerization can be determined in this case from time resolved emission measurements. 
Fig. 3. Projection on the a, b crystallographic plane of a thioindigo molecule doped substitutionally into a benzoic acid crystal. Two of the four tautomer configurations of the two equivalent acid dimers sandwiching the indigo molecule are shown.

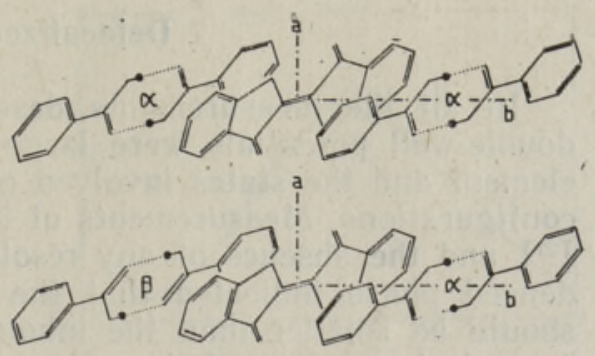

These measurements lead therefore to a straighforward determination of the rate of tautomerization. The situation illustrated in Fig. 2 is realized in thio- and selenoindigo-doped benzoic acid crystals. As both the crystal and the dopant are centrosymmetric, there are actually two equivalent acid dimers separated by two lattice spacings coupled to one impurity molecule and four interconverting tautomer configurations that have to be considered (see Fig. 3). Two of these are centrosymmetric, the energy degeneracy of the two other polar configurations can be lifted in an applied electric field [ $\left.{ }^{21}\right]$. The measurements on thioindigo in benzoic acid are shown in Fig. 4. The fit of all experimental data could be obtained with just two adjustable parameters, the rate of tautomerization and the lifetime of the excited state of thioindigo $\left[{ }^{23}\right]$. Within experimental accuracy the intrinsic lifetine of the impurity is not affected by the detailed structure of the environment and the tautomerization of the acid dimers on either side of the impurity occurs in an uncorrelated fashion and with the same rate. Fig. 4. also illustrates the large deuteration effect: in the deuterated acid nearly all emission occurs from the initially excited level because the rate of tautomerization is reduced by about three orders of magnitude. Measurements of this kind have been performed for a variety of systems and the rate of tautomerization was found to be remarkably independent of the nature of the impurity and the magnitude of the asymmetry of the double-well: its value is $2-4 \cdot 10^{8} \mathrm{~s}^{-1}$.

Fig. 4. Top: emission spectra of thioindigo in benzoic acid recorded under selective excitation of the tautomer configuration with the highest (lowest) energy when thioindigo is in the excited (ground) electronic state. The lines marked $R_{1}, R_{2}$ and $R_{3}$ correspond to the different tautomer configurations $|\alpha, \alpha\rangle,|\alpha, \beta\rangle$ and $|\beta, \alpha\rangle,|\beta, \beta\rangle$. The bands marked $p h$ and $v$ are phonon side bands and vibronic lines, respectively some of the other sharp lines are Raman transitions of the benzoic acid matrix. Bottom: time resolved fluorescence emission from the $R_{1}, R_{2}$ and $R_{3}$ configurations subsequent to selective excitation of $R_{1}$.

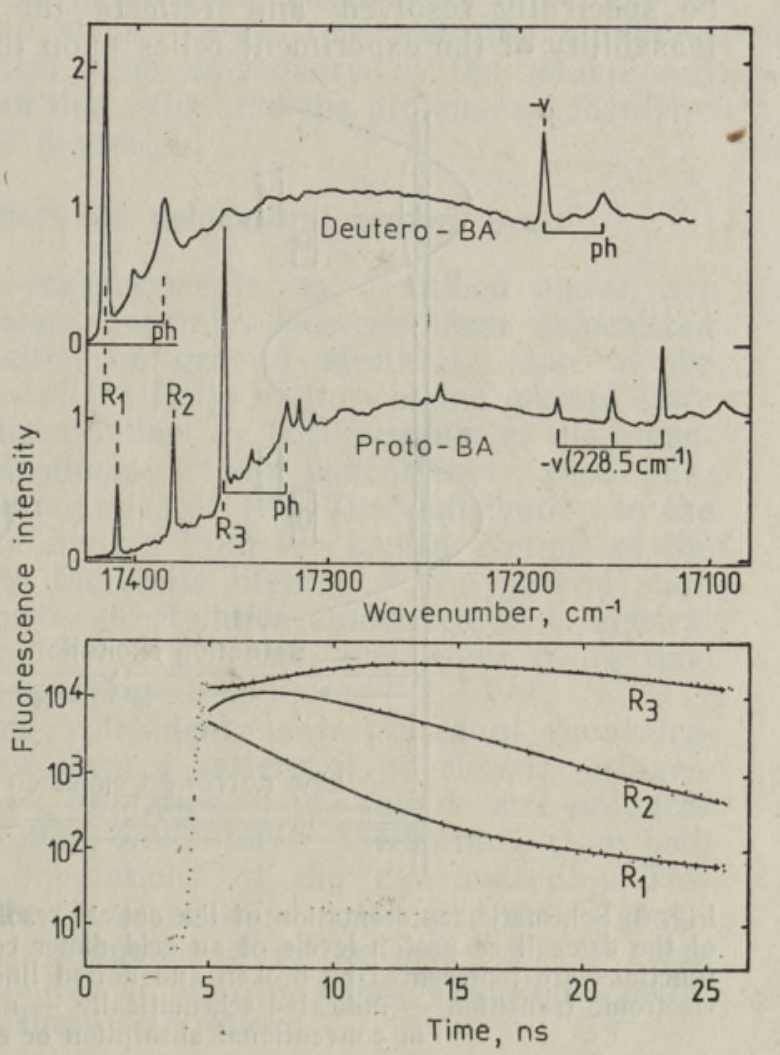




\section{Delocalized proton levels}

In all the measurements described above the asymmetries of the double-well potentials were large as compared to the tunneling matrix element and the states involved correspond therefore to localized proton configurations. Measurements of benzoic acid dimers in the vapor phase $\left[{ }^{27}\right]$ and the absence of any resolved tunneling substructure in the condensed phase indicated that the magnitude of the tunneling splitting should be smaller than the inhomogeneous linewidth, i.e. less than ca. $1 \mathrm{~cm}^{-1}$. A measure of the value of the tunnelig matrix element and the relaxation dynamics between delocalized proton levels is of particular interest and becomes possible in a system where the assymmetry of the double-well potential is accidentally nearly zero when the dye impurity is unexcited while it becomes large for the acid dimers next to the excited dye.

\section{Resolution of the tunneling splitting by fluorescence line narrowing}

The situation described above is illustrated in Fig. 5. The ground state splitting of the delocalized levels is not resolved in conventional absorption and emission spectra, and was inferred from the temperature dependence of the absorption line intensities of the transitions to the different localized excited states. Fluorescence line narrowing techniques on the other hand are capable to resolve the ground state splitting: narrowband laser excitation transfers population from the ground state levels into the excited state for those molecules for which the transition energy in the inhomogeneous distribution matches the laser frequency. After relaxation by tautomerization the resulting emission can be spectrally resolved and reflects the ground state splittings. The feasability of the experiment relies upon the fact that the inhomogeneous

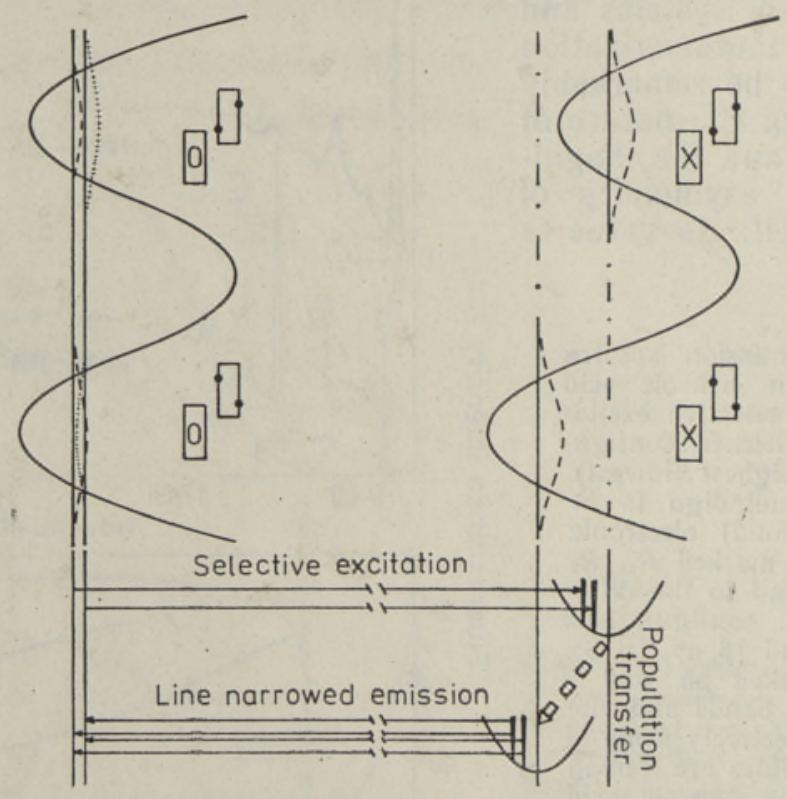

Fig. 5. Schematic representation of the optical resolution by fluorescence line narrowing of the delocalized proton levels of an acid dimer coupled to a dye molecule. The wavefunctions are represented by broken and dotted lines. The inhomogeneous width of the electronic transition - indicated schematically - masks the ground state level splitting 
Fig. 6. Line narrowed emission spectra obtained for thioindigo in benzoic acid: excitation into a vibrational level (top) and the 0 -0-transition (bottom) of the higher energy $|\alpha, \beta\rangle,|\beta, \alpha\rangle$ configuration. The homogeneous linewidth of the transitions in the excitation process masks the ground state splitings when a vibrational level is involved, while it makes a negligeable contribution to the instument limited linewidth of the bottom spectrum obtained by excitation of the 0 -0-transition.

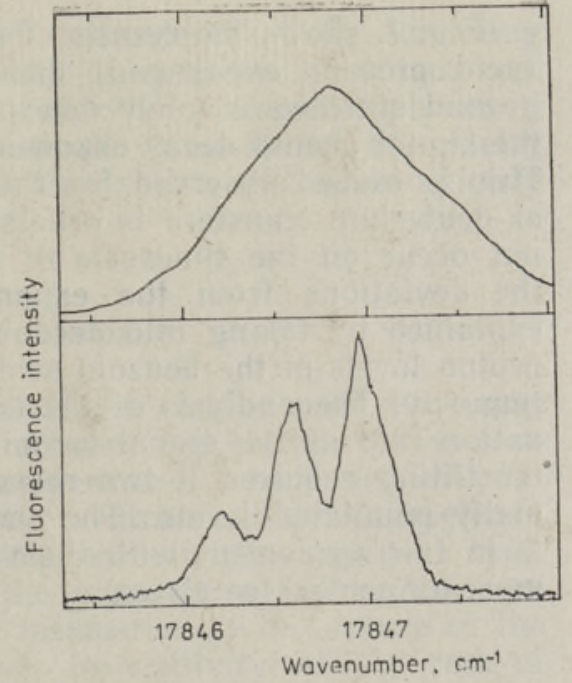

distributions of the transitions involved are correlated. The actual experiment $\left.{ }^{26}\right]$ was done with thioindigo in benzoic acid (a different substitutional site than the one involved in the time resolved experiment described above), and shows a somewhat more complex structure as again two-coupled acid dimers have to be considered. The resulting line narrowed spectrum is shown in Fig. 6. The residual linewidth is instrument-limited; any broadening due to incomplete correlations of the inhomogeneous broadenings is smaller than about $0.1 \mathrm{~cm}^{-1}$ : this is a remarkable result as the excited states involved in the transitions correspond to polar and nonpolar tautomer configurations, respectively. The value of the tunneling matrix element obtained in these experiments is $0.16 \pm 0.01 \mathrm{~cm}^{-1}$ (this value equals one half the tunneling splitting of a symmetric dimer). The asymmetry of the double-well potential is slightly smaller than this value and the protons are therefore delocalized to a very significant degree.

\section{Relaxation dynamics between delocalized proton levels}

Time resolved fluorescence measurements, as described above, are inadequate to acces the relaxation dynamics between these delocalized levels which are associated with the ground electronic state of the impurity. An indirect evaluation of the finite lifetime of the ground state levels was obtained from a determination, by hole-burning, of the homogeneous linewidth of the transitions at low temepatures when pure dephasing can considered to be negligeable $\left[{ }^{20}\right]$. The contribution to the linewidth in excess of the term coming from the known lifetime of the excited state was attributed to the finite lifetime of the ground state levels. In view of the well-known uncertainties associated with spectral hole-burning techniques a more direct measure was made using time resolved picosecond transient grating techniques.

In these experiments two time coincident laser pulses of equal frequency cross in the sample, and form a pattern of interference fringes. This leads to a spatially periodic excitation of the sample and produces variations of the index of refraction, which have contributions from both the excited and ground state populations of the dye molecules. This index grating is probed by a time delayed third pulse, and the intensity of the diffracted signal, which is proportional to the square of the index variations, is measured as a function of the time delay. The signal therefore maps the time evolution of these populations. 
Fig. 7. shows the results of such measurements for proto and deutero (acid protons exchanged) benzoic acid [25]. If the recovery of the ground state were solely determined by the lifetime of the excited state the signal should decay exponentially with half the excited state lifetime. This is indeed observed for the deuterated compound, because the rate of deuterium transfers is orders of magnitude slower and does virtually not occur on the timescale of the measurement. In proto benzoic acid the deviations from the exponential decay are obvious and can be explained by taking into account the relaxation between the delocalized proton levels of the benzoic, acid dimers near the ground state thioindigo impurity. The analysis of the data was made introducing a single relaxation rate as the signal to noise ratio did not warrant a multiparameter fitting required if two relaxation rates are used for the three thermally populated levels. The value obtained for this rate $\left(3.5 \cdot 10^{8} \mathrm{~s}^{-1}\right)$ is in fair agreement with the value obtained from the optical linewidth measurements (see above).

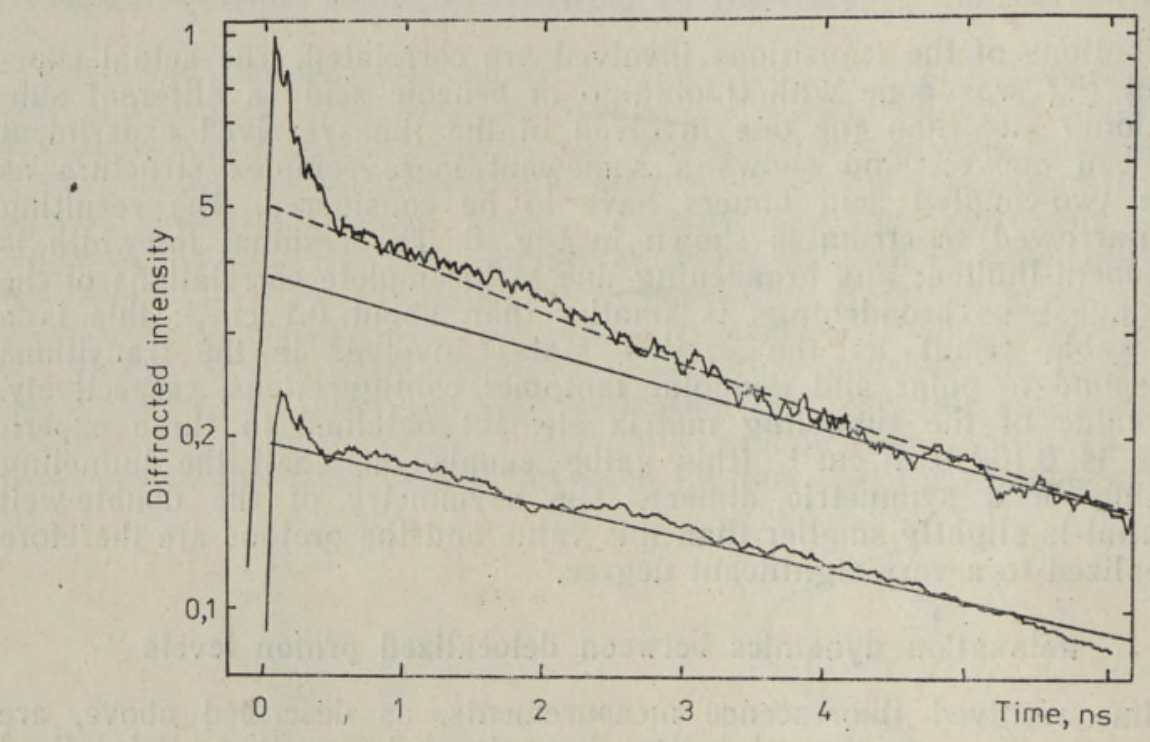

Fig. 7. Transient grating measurements in proto (top) and deutero (bottom) benzoic acid doped with thioindigo. The full and broken lines are calculated decays neglecting and including relaxation processes of benzoic acid dimers near ground state thioindigo molecules. The peak near $t=0$ reflects a fast process possibly related to multiphoton excitation of higher electronic states

Relaxation processes in double-well potentials can be related to modulations by phonons of the asymmetry and the barrier, i.e. the tunneling matrix element. Using Fermis golden rule and taking the phonon density of states to be proportional to the square of the energy, it is easy to see that rate of population relaxation is proportional to the square of the sum of two terms: one is the product of the tunneling matrix element and the modulations of the asymmetry, the second is the product of the asymmetry and the modulations of the tunneling matrix element $\left[{ }^{22}\right]$. Our results indicate that this rate is quite insensitive to the absolute value of the asymmetry and suggest therefore that the first term dominates: the measured relaxation rates should therefore be proportional to the square of the tunneling matrix element. The deute- 
ration effect on these rates of about $10^{+3}$ indicates that the tunneling matrix element in deuterated benzoic acid is reduced by a factor of about 30 and is of the order of $5 \cdot 10^{-3} \mathrm{~cm}^{-1}$ : in condensed phases it will be very difficult to measure such a small value as the asymmetry is likely to be always much greater and the system will be completely localized.

\section{Summary and conclusion}

We have shown here how optical techniques can be used to assess the level structure and the dynamics of a tunneling system in the condensed phase in the limit of very low temperatures where conventional nuclear magnetic resonance and inelastic neutron scattering methods become inoperative. Our results for benzoic acid dimers should be typical for carboxylic acid dimers, because the parameters determined here with different probe molecules are fairly insensitive to the nature of the probe. One unexpected result is the great insensitivity of the rate of relaxation to the asymmetry of the double-well potential. This point certainly deserves further investigation and confirmation.

\section{Acknowledgements}

This paper has been given at the all-union symposium on Modern Methods of Laser Spectroscopy of Molecules in Low-Temperature Media. The author would like to express his sincere gratitude to the hosts of the Estonian Academy of Sciences for all the efforts that have made this visit to Tallinn and Tartu possible and very stimulating scientifically and enjoyable on a personal level.

\section{REFERENCES}

1. Spectroscopy and Excitation Dynamics of Condensed Molecular Systems (eds V. M. Agranovich, R. M. Hochstrasser). Amsterdam, North Holland, 1983; see I. S. Osad'ko, p. 437, G. J. Small, p. 515, R. I. Personov, p. 555.

2. Friedrich, J., Haarer, D. Angew. Chem. (Int. Ed.), 23, 113 (1984).

3. Fearey, B. L., Stout, R. P., Hayes, J. M., Small, G. J. J. Chem. Phys., 78, 7013 (1983).

4. Breinl, W., Friedrich, J., Haarer, D. Chem. Phys. Lett., 106, 487 (1984).

5. Silbey, R., Harris, R. A. J. Chem. Phys., 80, 2615 (1984).

6. Harris, R. A., Silbey, R. J. Chem. Phys., 83. 1096 (1985).

7. Parris, P. E., Silbey, R. J. Chem. Phys., 83, 5619 (1985).

8. Carmeli, B., Chandler, D. J. Chem. Phys., 82, 3400 (1985).

9. Graf, F., Meyer, R., Ha, T.-K., Ernst, R. J. Chem. Phys., 75, 2914 (1981).

10. Nagaoka, S., Hirota, N., Matsushita, T., Nishimoto, K. Chem. Phys. Lett., 92, 498 (1982)

11. Hayashi, S., Umemura, J., Kato, S., Morokuma, K. J. Phys. Chem., 88, 1330 (1984).

12. Meyer, R., Ernst, R. R. J. Chem. Phys., 86, 784 (1987).

13. Nagaoka, S., Terao, T., Imashiro, F., Saika, A., Hirota, N., Hayashi, S. Chem. Phys. Lett., 80, 580 (1981).

14. Nagaoka, S., Terao, T., Imashiro, F., Saika, A., Hirota, N., Hayashi, S. J. Chem. Phys., 79, 4694 (1983).

15. Meier, B. H., Graf, E., Ernst, R. R. J. Chem. Phys., 76, 767 (1982).

16. Meier, B. H., Meyer, R., Ernst, R. R., Stöckli, A., Furrer, A., Hälg, W., Anderson, I. Chem. Phys. Lett., 108, 522 (1984).

17. Meier, B. H., Meyer, R., Ernst, R. R., Zolliker, P., Furrer, A., Hätg, W. Chem. Phys. Lett., 103, 169 (1983). 
18. Fischer, P., Zolliker, P., Meier, B. H., Ernst, R. R., Hewat, A. W., Jorgensen, D., Rotella, J. J. J. Solid State Chem., 61, 109 (1986).

19. Meier, B. H., Ph. D. Thesis, ETH, Nr. 7620, Zürich, 1984.

20. Clemens, J. M., Hochstrasser, R. M., Trommsdorff, H. P. J. Chem. Phys., 80, 1744 (1984).

21. Trommsdorff. H. P., Casalegno, R., Miller. R. J. D., Clemens, J. M., Hochstrasser, R. M. J. Luminescence, 31/32, 517 (1984).

22. Trommsdorff, H. P. In: Tunneling (eds J. Jortner and B. Pullman). Dordrecht, D. Reidel, 1986, 103.

23. Holtom, G. R., Hochstrasser, R. M., Trommsdorff, H. P. Chem. Phys. Lett., 131, 44 (1986).

24. Pierre, M., Trommsdorff, H. P. In: Dynamics of Molecular Crystals (ed. J. Lascombe). Amsterdam, Elsevier, 1987, 519.

25. Pierre, M., Trommsdorff, H. P., Hochstrasser, R. M. In: Quantum Aspects of Molecular Motions in Solids, v. 17 (eds A. Heidemann et al.). Berlin, Springer, $1987,186$.

26. Hochstrasser, R. M., Trommsdorff, H. P. J. Chem. Phys. (in press).

27. Poeltl, D. E., McVey, J. K., J. Chem. Phys., 78, 4349 (1983); J. Chem. Phys., 80, 1801 (1984).

Univesrity of Science, Technology and Medicine of Grenoble, France

Received

Sept. 21,1987

\section{H. P. TROMMSDORFF}

\section{OPTILISED SIIRDED PROOTONULEKANDESUSTEEMIDES}

Karboksüülhappe kristallidesse viidud asenduslisandi optiliste tsentrite kitsajoonelised spektrid sisaldavad madalatel temperatuuridel teavet ümbruse prootonstruktuuri kohta. Seda asjaolu kasutati tio- ja selenoindigot sisaldavates bensoehappe kristallides happedimeeride tautomerisatsiooni dünaamika mõōtmiseks ja seda prootonülekannet juhtiva kahenõolise potentsiaali tunnelmaatrikselemendi määramiseks.

\section{Г. П. ТРОММСДОРФ}

\section{ОПТИЧЕСКИЕ ПЕРЕХОДЫ В СИСТЕМАХ С ПЕРЕНОСОМ ПРОТОНА}

Узкополосные низкотемпературные спектры оптических примесных центров замещения в кристаллах карбоксильной кислоты несут информацию о. протонной структуре окружения. Этот факт был использован для изучения кристаллов бензойной кислоты, содержащих тио- и селеноиндиго, с целью измерения таутомерной динамики димеров кислоты и определения туннельного матричного элемента двухъямного потенциала, определяющего перенос протона. 